\title{
Excess Galactic Molecular Absorption Toward the Radio Galaxy 3C 111
}

\author{
F. Tombesi ${ }^{1,2,3}$ C. S. Reynolds ${ }^{2}$, R. F. Mushotzky ${ }^{2}$, and E. Behar ${ }^{4}$ \\ ${ }^{1}$ X-Ray Astrophysics Laboratory, NASA/Goddard Space Flight Center, Greenbelt, MD 20771, USA; francesco.tombesi@nasa.gov \\ ${ }^{2}$ Department of Astronomy, University of Maryland, College Park, MD 20742, USA; ftombesi@astro.umd.edu \\ ${ }^{3}$ Department of Physics, University of Rome "Tor Vergata", Via della Ricerca Scientifica 1, I-00133 Rome, Italy; francesco.tombesi@roma2.infn.it \\ ${ }^{4}$ Department of Physics, Technion 32000, Haifa 32000, Israel \\ Received 2017 February 13; revised 2017 April 28; accepted 2017 May 4; published 2017 June 14
}

\begin{abstract}
We show the combined spectral analysis of Chandra high-energy transmission grating and XMM-Newton reflection-grating spectrometer observations of the broad-line radio galaxy $3 \mathrm{C} 111$. This source is known to show excess neutral absorption with respect to the one estimated from $21 \mathrm{~cm}$ radio surveys of atomic $\mathrm{H}$ I in the Galaxy. However, previous works were not able to constrain the origin of such an absorber as local to our Milky Way or intrinsic to the source $(z=0.0485)$. The high signal-to-noise grating spectra allow us to constrain the excess absorption as being due to intervening gas in the Milky Way, and we estimate a time-averaged total column density of $N_{\mathrm{H}}=(7.4 \pm 0.1) \times 10^{21} \mathrm{~cm}^{-2}$, a factor of two higher than the tabulated $\mathrm{H}$ I value. We recommend using the total average Galactic column density estimated here when studying 3C 111 . The origin of the extra Galactic absorption of $N_{\mathrm{H}}=4.4 \times 10^{21} \mathrm{~cm}^{-2}$ is likely due to molecular gas associated with the Taurus molecular cloud complex toward 3C 111, which is our nearest star-forming region. We also detect a weak $(\mathrm{EW}=16 \pm 10 \mathrm{eV})$ and narrow (FWMH $<5500 \mathrm{~km} \mathrm{~s}^{-1}$, consistent with optical $\mathrm{H} \alpha$ ) Fe $\mathrm{K} \alpha$ emission line at $E=6.4 \mathrm{keV}$, likely from the torus in the central regions of 3C 111, and we place an upper limit on the column density of a possible intrinsic warm absorber of $N_{\mathrm{H}}<2.5 \times 10^{20} \mathrm{~cm}^{-2}$. These complexities make 3C 111 a very promising object for studying both the intrinsic properties of this active radio galaxy and the Galactic interstellar medium, if used as a background source.
\end{abstract}

Key words: galaxies: active - local interstellar matter - X-rays: galaxies - X-rays: ISM

\section{Introduction}

Active galactic nuclei (AGN) are classified as radio-loud and radio-quiet, depending on their radio luminosity with respect to the optical (Kellermann et al. 1989). The origin of this dichotomy is still not known, but it is manifested by the presence of powerful, often relativistic, radio jets in radio-loud AGN (e.g., Urry \& Padovani 1995). On the other hand, radioquiet AGN, most notably Seyfert galaxies and quasars, show clear features of massive, sub-relativistic winds (e.g., Tombesi et al. 2010a; Gofford et al. 2015). However, winds have been recently reported in radio-loud sources as well (e.g., Reeves et al. 2009; Tombesi et al. 2010b, 2014; Torresi et al. 2010, 2012; Gofford et al. 2015).

The X-ray band is particularly promising for investigating the possible origin of this dichotomy, because the radiation is emitted close to the central supermassive black hole (SMBH), and can therefore retain information from both the accretion disk and intervening absorption and emission in the regions surrounding the AGN or the host galaxy interstellar medium (ISM; e.g., Sambruna et al. 1999, 2011; Lewis et al. 2005; Ogle et al. 2005; Ballo et al. 2011; Braito et al. 2011; Tombesi et al. 2011, 2016; Lohfink et al. 2015). Coordinated observations in the X-ray, optical, and radio also facilitated the study of the connection between the disk, jet, and winds in some sources (e.g., Chatterjee et al. 2011; Tombesi et al. 2012; Lohfink et al. 2013).

Here, we report on the analysis of a long $150 \mathrm{ks}$ Chandra HETG observation of the broad-line radio galaxy (BLRG) 3C $111(z=0.0485)$, in combination with archived XMMNewton reflection-grating spectrometer (RGS) spectra. This is the third paper of this series; the previous two focused on 3C 390.3 and 3C 120, respectively (Tombesi et al. 2016, 2017).
The radio galaxy $3 \mathrm{C} 111$ is an X-ray bright BLRG and it is classified as a Fanaroff-Riley type II (FRII) source with a double-lobe/single-jet morphology (Linfield \& Perley 1984). The inner jet displays superluminal motion (Vermeulen \& Cohen 1994; Chatterjee et al. 2011). This source was also detected in the $\gamma$-ray band with Fermi (Kataoka et al. 2011; Grandi et al. 2012).

The main objective of the Chandra HETG campaign was to study the possible X-ray warm absorbers in the brightest radio galaxies. However, we do not find significant evidence for an intrinsic soft X-ray ionized absorber in this source. The apparent lack of a warm absorber is puzzling, due to the fact that $3 \mathrm{C} 111$ shows powerful jets and accretion disk winds driven by the SMBH (e.g., Tombesi et al. 2010b; Chatterjee et al. 2011). Moreover, warm absorbers have been detected in most bright radio galaxies (e.g., Reeves et al. 2009; Torresi et al. 2010, 2012; Braito et al. 2011). On the other hand, this source is known to show an excess of cold/neutral absorption with respect to the value estimated from $21 \mathrm{~cm}$ radio surveys of atomic HI. Previous X-ray studies have not been able to constrain the origin of such an absorber as local to our Milky Way or intrinsic to 3C 111 because the redshift could not be constrained (e.g., Reynolds et al. 1998; Lewis et al. 2005; Ballo et al. 2011; Tombesi et al. 2013).

We note that 3C 111, along with other bright AGN, has been used as an extragalactic background radio source to explore complex atomic and molecular gas regions in the Milky Way (e.g., Marscher et al. 1993; Moore \& Marscher 1995). In fact, 3C 111 is located at a relatively low latitude with respect to the Galactic plane and it lies behind the giant Taurus molecular cloud, which is the nearest large star-forming region in our Galaxy (Ungerer et al. 1985; Ungerechts \& Thaddeus 1987). 
Several detailed studies of the structure in the atomic component of the ISM have suggested that complexities exist on scales as small as a few tens of au. The first indication that such small-scale H I structures may exist was reported by Dieter et al. (1976). Using VLBI techniques to measure changes in the visibility function in the Galactic $\mathrm{H}$ I absorption line toward 3C 147, they proposed that the line variations were due to a small cloud with a dimension of about 70 au and a density of $\simeq 10^{5}$ atoms $\mathrm{cm}^{-3}$.

Moreover, Faison et al. (1998) used VLBA and the VLA to image Galactic H I in absorption in the direction of three other bright extragalactic sources, namely 3C 138, 2255+416, and CJ1 0404+768. They suggested that the small-scale opacity structures seen toward 3C 138 and $2255+416$ may be due to density variations, spin temperature variations, velocity turbulence in the atomic gas, or a combination of these effects. If the opacity variations are due to fluctuations in density, then they would suggest clouds with high densities of $\sim 10^{6} \mathrm{~cm}^{-3}$ in the cold neutral medium on $\sim 10$ au scales.

Aside from atomic $\mathrm{HI}$, there has also been evidence for small-scale structures in the diffuse molecular gas in our Galaxy. For instance, Moore \& Marscher (1995) reported changes in the column density of formaldehyde $\left(\mathrm{H}_{2} \mathrm{CO}\right)$ toward the compact sources NRAO 150, 3C 111, and BL Lac. They observed the three sources for over three years with the VLA in the $6 \mathrm{~cm} \mathrm{H}_{2} \mathrm{CO}$ line. The motion of the sources due to parallax, as well as the proper motions of the absorbing gas, caused the relative line of sight through the molecular gas to the extragalactic sources to change with time. They observed significant variations in the molecular column density toward NRAO 150 and 3C 111, which indicate structures on the scales of $\sim 10$ au and densities of $\sim 10^{6} \mathrm{~cm}^{-3}$. Several other studies have been performed in order to map the molecular $\mathrm{OH}$ and $\mathrm{CO}$ distribution in the Galaxy using compact background continuum sources in the radio and mm-waves (e.g., Liszt \& Lucas 1996, 1998).

In the X-ray band, compact X-ray binaries have provided important information regarding the composition and ionization of the Galactic ISM through absorption, although such sources are mostly distributed along the galactic plane (e.g., Schulz et al. 2002; Gatuzz et al. 2015). Some attempts to use bright extragalactic continuum sources to explore our Galaxy multiphase and multiscale ISM along several lines of sight have also been reported in the UV and X-ray bands, for instance recently in the context of the origin of the Fermi bubbles (e.g., Fox et al. 2015; Nicastro et al. 2016; Bordoloi et al. 2017).

A complementary method to explore the atomic and molecular hydrogen content from many different sightlines in the Milky Way is provided by gamma-ray burst (GRB) afterglows in the X-ray band. We note that the value reported for the cold absorption toward 3C 111 is about a factor of two higher than the upper limit reported by Willingale et al. (2013) for GRB sightlines.

\section{Data Analysis and Results}

We describe the analysis of the Chandra HETG spectrum of the broad-line radio galaxy 3C 111. It was observed on 2014 November 4 for a single exposure of $143 \mathrm{ks}$ (ID 16219). The spectrum was extracted using the $C I A O$ package v4.7. Only the first order of dispersed spectra were considered for both the medium energy grating (MEG) and high-energy grating (HEG), and the \pm 1 orders for each grating were subsequently

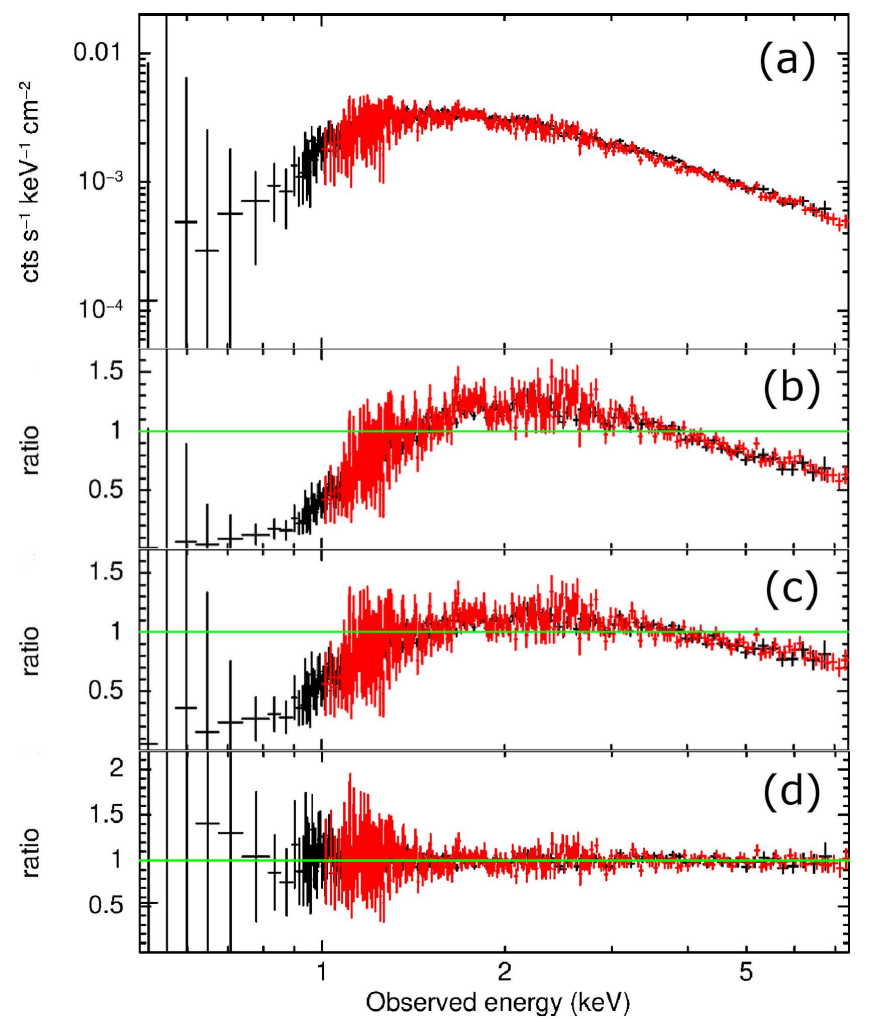

Figure 1. Chandra MEG (black) and HEG (red) spectra of 3C 111. The data are binned to four times the FWHM resolution and to a minimum signal-tonoise of 2 in order to emphasize the broadband curvature of the spectrum due to the absorber column density. Panel (a): MEG and HEG spectra. Panel (b): ratio with respect to a power-law continuum model. Panel (c): ratio with respect to a power-law continuum model including the tabulated Galactic absorption value of $N_{\mathrm{H}}=3 \times 10^{21} \mathrm{~cm}^{-2}$. Panel (d): ratio with respect to the best-fit model consisting of a power-law continuum, Galactic absorption with free column density, and an $\mathrm{Fe} \mathrm{K} \alpha$ emission line.

combined for each sequence. The background count rate is found to be negligible. The resultant spectra were binned to the FWHM of their spectral resolution, which corresponds to $\Delta \lambda=0.023 \AA$ and $\Delta \lambda=0.012 \AA$ bins for MEG and HEG, respectively. The MEG and HEG spectra were analyzed in the energy bands $E=0.5-7 \mathrm{keV}$ and $E=1-7.5 \mathrm{keV}$, respectively. The MEG and HEG count rates are $0.47 \mathrm{cts} \mathrm{s}^{-1}$ and 0.28 cts s $^{-1}$, respectively. The spectral analysis was performed using the software XSPEC v.12.8.2 and the C-statistic was applied. We performed simultaneous fits of the MEG and HEG spectra considering a free cross-normalization constant, with results always being very close to unity. All parameters are given in the source rest frame and the errors and limits are at the $90 \%$ level if not otherwise stated. Standard Solar abundances are assumed (Asplund et al. 2009).

The MEG and HEG spectra of 3C 111 are shown in panel a of Figure 1. We started the spectral modeling using a powerlaw continuum with photon index $\Gamma \simeq 0.9$. As can be seen from panel (b) of Figure 1, this does not provide a good fit $(C / u=8182 / 2220)$, with the soft X-ray residuals and very flat spectral index indicating the requirement for a neutral absorption component.

We then included a neutral absorption component modeled with tbabs in XSPEC. This model calculates the cross-section for X-ray absorption by the ISM as the sum of the crosssections for X-ray absorption due to the gas-phase, the grainphase, and the molecules (Wilms et al. 2000). 
We start using a column density of $N_{\mathrm{H}}=3 \times 10^{21} \mathrm{~cm}^{-2}$ derived from NASA's HEASARC $n h$ tool. ${ }^{5}$ This is the intermediate value between the weighted average values estimated by the Leiden/Argentine/Bonn Survey of Galactic H I (Kalberla et al. 2005) of $N_{\mathrm{H}}=2.91 \times 10^{21} \mathrm{~cm}^{-1}$ and by the Dickey \& Lockman $\mathrm{HI}$ in the Galaxy (Dickey \& Lockman 1990) of $N_{\mathrm{H}}=3.15 \times 10^{21} \mathrm{~cm}^{-2}$. This fit provides a high statistical improvement with $C / u=4145 / 2220$ and a less-flat power-law photon index of $\Gamma \simeq 1.2$. The ratio of the spectra with respect to the power-law, including the tabulated Galactic absorption, is shown in panel (c) of Figure 1. We note an improvement with respect to panel (b), but the fit is still not satisfactory.

Previous observations of the radio galaxy 3C 111 showed an excess absorption with respect to the tabulated Galactic value of $N_{\mathrm{H}} \sim 5 \times 10^{21} \mathrm{~cm}^{-2}$. However, it was still not possible to constrain the origin of such an absorber as local to our Milky Way or intrinsic to 3C 111 because the redshift could not be constrained (e.g., Reynolds et al. 1998; Lewis et al. 2005; Ballo et al. 2011; Tombesi et al. 2013).

We then included a new ztbabs absorption component assuming the redshift of the source. We obtained a high-fit improvement with $C / u=2264 / 2219$ for a column density of $N_{\mathrm{H}}=$ $(5.1 \pm 0.3) \times 10^{21} \mathrm{~cm}^{-2}$ and a photon index of $\Gamma \simeq 1.6$. We then left the redshift free to vary and we obtained an additional very high-fit improvement of $\Delta C / \Delta u=25 / 1$, corresponding to a statistical confidence level of $5 \sigma$. The best-fit redshift is $z=-0.002_{-0.038}^{+0.002}$, which is consistent with being local to our Milky Way galaxy, instead of 3C 111 at a redshift of $z=0.0485$. Considering a single neutral ztbabs absorber component to model both the tabulated and excess absorption, we obtained a column density of $N_{\mathrm{H}}=(7.5 \pm 0.2) \times 10^{21} \mathrm{~cm}^{-2}$ and a better-constrained redshift value of $z=-0.002_{-0.008}^{+0.002}$. The contour plots of these parameters are shown subsequently in Figure 4.

The main spectral features responsible for the absorption are the photoelectric edges from $\mathrm{O}$ I K at $E=0.543 \mathrm{keV}, \mathrm{Fe} \mathrm{I} \mathrm{L}_{1}$ at $E=0.845 \mathrm{keV}$, Fe I $\mathrm{L}_{2}$ at $E=0.72 \mathrm{keV}$, and $\mathrm{NeI} \mathrm{K}$ at $E=0.87 \mathrm{keV}$. In particular, because the $\mathrm{O}$ edge is so deep, the fit is mostly driven by counts in the latter three edges. In fact, we obtain the same results when restricting the fit to energies higher than $E=0.7 \mathrm{keV}$. Moreover, we note that the $\mathrm{Fe} \mathrm{I} \mathrm{L}_{1}$ edge is significantly weaker than the $\mathrm{Fe} \mathrm{I} \mathrm{L}_{2}$ edge, and therefore it will only marginally affect the fit (e.g., Schulz et al. 2002). We checked that we had obtained the same results using different absorption models in XSPEC, such as phabs and $w a b s$, or the detailed X-ray absorption code tbnew_gas (Wilms et al. 2000), which is still a beta-test version. ${ }^{6}$

Looking at the ratio between the data and this model zoomed in to the $E=6-7 \mathrm{keV}$ band in Figure 2, we observe the presence of a faint emission line at the expected energy of the Fe $\mathrm{K} \alpha$ fluorescence emission line of $E=6.4 \mathrm{keV}$. Therefore, we consider the final best-fit model to be composed of a powerlaw continuum, a single Galactic neutral absorber, and an $\mathrm{Fe}$ $\mathrm{K} \alpha$ Gaussian emission line. The ratio of the data with respect to the best-fit model is shown in panel (d) of Figure 1.

Assuming a redshift of zero, i.e., local to our Milky Way, the best-fit parameters are a column density of $N_{\mathrm{H}}=(7.7 \pm 0.1) \times$ $10^{21} \mathrm{~cm}^{-2}$, a power-law photon index of $\Gamma=1.62 \pm 0.02$, and an emission line at the energy of $E=6.41 \pm 0.03 \mathrm{keV}$ with

\footnotetext{
5 http://heasarc.gsfc.nasa.gov/cgi-bin/Tools/w3nh/w3nh.pl

6 http://pulsar.sternwarte.uni-erlangen.de/wilms/research/tbabs/
}

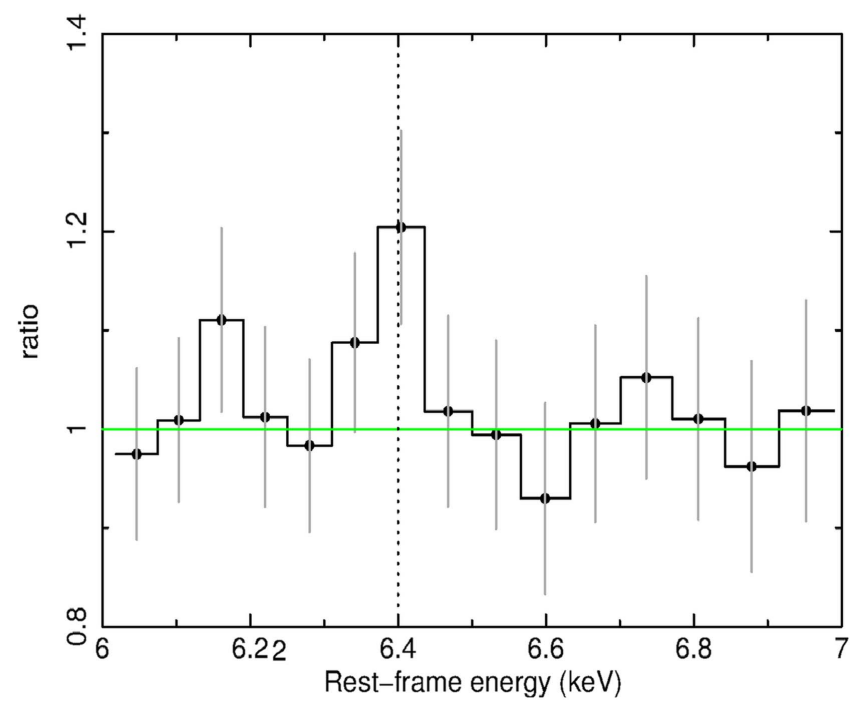

Figure 2. Ratio between the Chandra HEG spectrum and an absorbed powerlaw model zoomed in to the energy band $E=6-7 \mathrm{keV}$. The vertical dotted line indicates the $\mathrm{Fe} \mathrm{K} \alpha$ line at the energy of $E=6.4 \mathrm{keV}$. The data are binned to two times the FWHM resolution for clarity.

intensity $I=(1.2 \pm 0.7) \times 10^{-5} \mathrm{ph} \mathrm{s}^{-1} \mathrm{~cm}^{-2}$, width $\sigma<$ $50 \mathrm{eV}$, and equivalent width $\mathrm{EW}=16 \pm 10 \mathrm{eV}$. The bestfit statistics is $C / u=2232 / 2216$. The extrapolated absorption corrected fluxes in the energy intervals $E=0.5-2 \mathrm{keV}$ and $E=2-10 \mathrm{keV}$ are $3.0 \times 10^{-11} \mathrm{erg} \mathrm{s}^{-1} \mathrm{~cm}^{-2}$ and $6.1 \times$ $10^{-11} \mathrm{erg} \mathrm{s}^{-1} \mathrm{~cm}^{-2}$, respectively.

The intensity of the $\mathrm{Fe} \mathrm{K} \alpha$ emission line is consistent with previous estimates, and the low EW is consistent with the source being observed during a high state (Reynolds et al. 1998; Tombesi et al. 2010b, 2011, 2012, 2014; Ballo et al. 2011; Chatterjee et al. 2011). We note that the FWHM of the Fe K $\alpha$ of $\mathrm{FWMH}_{\mathrm{FeK}}<5500 \mathrm{~km} \mathrm{~s}^{-1}$ is consistent with the FWHM of the optical $\mathrm{H} \alpha$ line of $\mathrm{FWHM}_{\mathrm{H} \alpha}=4800 \pm 200 \mathrm{~km} \mathrm{~s}^{-1}$ (Eracleous $\&$ Halpern 2003). The full width at zero intensity (FWZI) from the $\mathrm{H} \alpha$ line is instead much broader FWZI $=18,400 \pm$ $3000 \mathrm{~km} \mathrm{~s}^{-1}$, but we cannot constrain this larger broadening if it is also present in the $\mathrm{Fe} \mathrm{K} \alpha$ line due to the limited signal-tonoise in the spectrum at these energies.

Using an XSTAR photoionized absorption table with turbulent velocity of $100 \mathrm{~km} \mathrm{~s}^{-1}$, and assuming the typical ionization of the warm absorber detected in other BLRGs of $\log \xi \simeq 2.5 \mathrm{erg} \mathrm{s}^{-1} \mathrm{~cm}$ and a velocity consistent with zero at the source rest frame (e.g., Reeves et al. 2009; Torresi et al. 2010, 2012), we estimate an upper limit of the column density of a possible warm absorber of $N_{\mathrm{H}}<2.5 \times 10^{20} \mathrm{~cm}^{-2}$. The fact that we do not clearly detect a warm absorber in this source could partially be due to the intervening absorption from our own Galaxy and/or to the fact that the ISM in this source could be hot, as observed in 3C 390.3 and 3C 120, and the low inclination estimated at $\simeq 18^{\circ}$ from the radio jet (e.g., Torresi et al. 2012; Tombesi et al. 2016, 2017).

\subsection{Combining Chandra and XMM-Newton Spectra}

3C 111 was observed twice with XMM-Newton, in 2001 for $45 \mathrm{ks}$ and in 2009 for $120 \mathrm{ks}$. Here, our focus is on the highenergy resolution RGS spectra in the energy band between $E=0.5-2.5 \mathrm{keV}$. We use both RGS1 and RGS2 detectors, and the latest pipeline data products. 


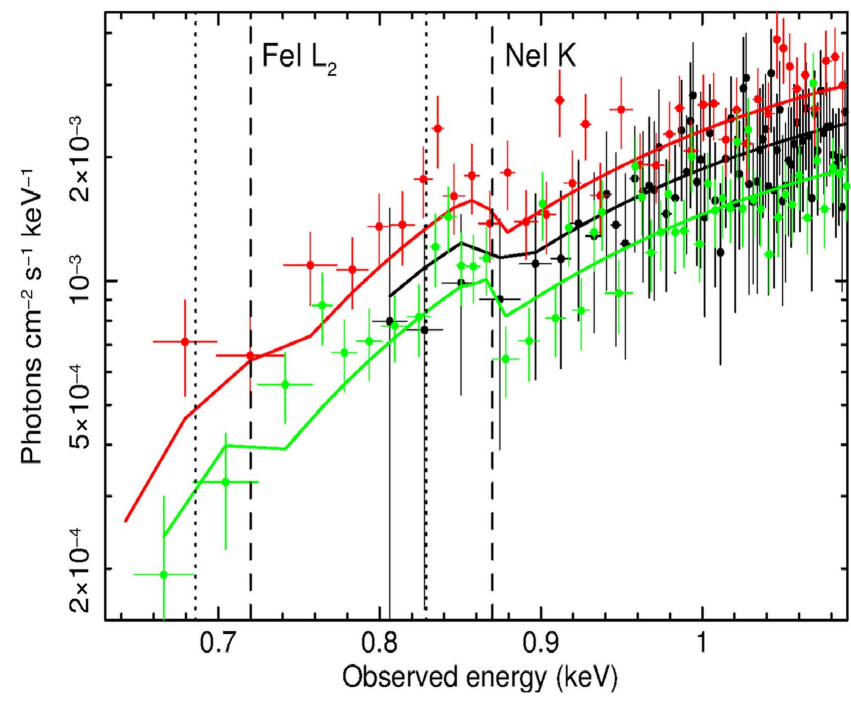

Figure 3. Grating spectra and best-fit absorbed power-law model zoomed in to the $E=0.6-1.1 \mathrm{keV}$ band. The Chandra MEG (black) is binned to two times the FWHM resolution and to a minimum signal-to-noise of 2 for clarity. The XMM-Newton RGS2 spectra taken in 2001 (red) and 2009 (green) are binned to a minimum signal-to-noise of 5 for clarity. The MEG data below $E=0.8 \mathrm{keV}$ are not shown here because the large error bars would make the comparison with the RGS less clear. The vertical dashed and dotted lines indicate the edge energies for $\mathrm{Fe} \mathrm{I}_{2}$ at $E=0.72 \mathrm{keV}$ and $\mathrm{Ne} \mathrm{I} \mathrm{K}$ at $E=0.87 \mathrm{keV}$ for a redshift of zero and intrinsic to $3 \mathrm{C} 111$, respectively.

First, we analyzed the RGS data alone using a model composed of a Galactic absorbed power-law continuum with extra cold absorption. Given the limited energy band of the RGS, we consider a power-law continuum of $\Gamma=1.6$ as estimated from previous broadband spectral analyses of these observations (Lewis et al. 2005; Ballo et al. 2011). Also in this case we find an extra absorption column density of $N_{\mathrm{H}} \simeq 5 \times 10^{21} \mathrm{~cm}^{-2}$, with a redshift consistent with zero with an uncertainty of $\Delta z \simeq 0.02$. Therefore, also the RGS data alone favor an absorber in the Milky Way, with a confidence level of $\sim 4 \sigma$.

We then performed a combined fit of the Chandra HETG and XMM-Newton RGS spectra, allowing for free power-law normalizations and cross-normalizations between observations and instruments, respectively. When fitting with a redshift fixed to the one of $3 \mathrm{C} 111(z=0.0485)$ or free to vary for the excess absorber, we find that a value consistent with zero is favored at $>6 \sigma(\Delta C / \Delta u=43 / 1)$.

Considering only one absorber, we estimate a power-law photon index of $\Gamma=1.60 \pm 0.01$, a total Galactic absorption column density of $N_{\mathrm{H}}=(7.4 \pm 0.1) \times 10^{21} \mathrm{~cm}^{-2}$, and a redshift of $z=-0.002_{-0.002}^{+0.003}$. We consider this total timeaveraged column density estimate to be the best-fit value from our analysis. The grating spectra and the best-fit absorbed power-law model zoomed in to the $E=0.6-1.1 \mathrm{keV}$ band for the Chandra MEG and the XMM-Newton RGS2 taken in 2001 and 2009 are shown in Figure 3. Instead, the contour plots of the column density with respect to the absorber redshift for the fits using the Chandra HETG and XMM-Newton RGS spectra alone, and both combined, are shown in Figure 4.

Given that the three observations were performed over a timescale of about a decade, we also performed a multi-epoch spectral fit, leaving the column density and redshift free to vary. We obtain that the redshift is always consistent with zero, but the column density shows a possible increase from

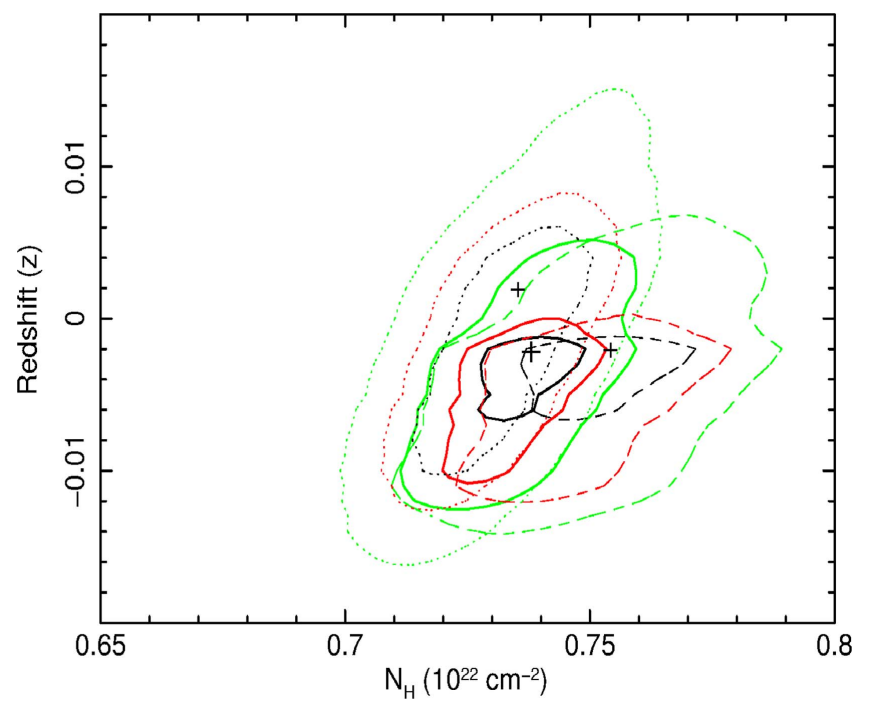

Figure 4. Contour plots comparing the column density with respect to the cosmological redshift of a single neutral absorber component modeling both the tabulated and the excess Galactic absorption. The contours refer to Chandra HETG (dashed), XMM-Newton RGS (dotted), and their combined fit (solid). The crosses indicate the best-fit values. The contours refer to confidence levels of $68 \%$ (black), $90 \%$ (red), and $99 \%$ (green), respectively.

$N_{\mathrm{H}}=(7.0 \pm 0.02) \times 10^{21} \mathrm{~cm}^{-2}$ for the XMM-Newton observation in 2001 to a value of $N_{\mathrm{H}}=(7.5 \pm 0.02) \times 10^{21} \mathrm{~cm}^{-2}$ for the XMM-Newton and Chandra observations of 2009 and 2014, respectively. Although very marginal, this increase in column density is in line with the reported temporal variability in the strength and profile of the $4.83 \mathrm{GHz} \mathrm{H}_{2} \mathrm{CO}$ absorption line in radio observations, which show that the cloud complex may contain inhomogeneities even on subparsec scales (e.g., Marscher et al. 1993; Moore \& Marscher 1995).

We also checked for variable $\mathrm{O}, \mathrm{Ne}$, and $\mathrm{Fe}$ abundances using the tbvarabs model in XSPEC (Wilms et al. 2000). The abundances are consistent with the Solar values within the uncertainties and we are able to place only lower limits of $A_{\mathrm{O}} \geqslant 0.5, A_{\mathrm{Ne}} \geqslant 0.9$, and $A_{\mathrm{Fe}} \geqslant 0.9$, respectively.

Finally, we performed a consistency check also analyzing the XMM-Newton EPIC-pn spectra simultaneous with the RGS observations performed in 2001 and 2009. The EPIC-pn has the highest sensitivity in the energy interval $E=0.5-10 \mathrm{keV}$. However, we note that the resolving power of the EPIC-pn of $E / \Delta E \simeq 5-10$ at the energies of $E=0.5-1.0 \mathrm{keV}$ is very limited compared to $E / \Delta E \simeq 300$ for the RGS and $E / \Delta E \simeq 600$ for the HETG, respectively. Moreover, there are significant cross-calibration uncertainties of both the shape and normalization of the effective area between the EPIC-pn and RGS.

We extracted the source and background spectra from 40 arcsec circular regions on the detectors and applied standard reduction techniques. We consider the absorbed power-law continuum model in the whole energy interval of $E=$ $0.5-10 \mathrm{keV}$. The column densities are estimated to be $N_{\mathrm{H}}=(0.61 \pm 0.04) \times 10^{21} \mathrm{~cm}^{-2}$ and $N_{\mathrm{H}}=(0.73 \pm 0.03) \times$ $10^{21} \mathrm{~cm}^{-2}$, respectively. We can place an upper limit on the redshift of the absorber of $z<0.025$, which is lower than the one of 3C 111 of $z=0.0485$. These values are in agreement with the much more accurate results derived from the analysis of the RGS and HETG spectra. 


\section{Discussion}

The radio galaxy 3C 111 was known from previous observations to show excess neutral absorption with respect to the column density of $N_{\mathrm{H}}=3 \times 10^{21} \mathrm{~cm}^{-2}$ estimated from $21 \mathrm{~cm}$ radio surveys of $\mathrm{HI}$ in the Galaxy (Dickey \& Lockman 1990; Kalberla et al. 2005). However, it was still not possible to constrain the origin of such an absorber as local to our Milky Way or intrinsic to 3C $111(z=0.0485)$ because the redshift of this component could not be constrained (e.g., Reynolds et al. 1998; Lewis et al. 2005; Ballo et al. 2011; Tombesi et al. 2013). The analysis of the Chandra HETG and XMMNewton RGS spectra of 3C 111 allowed us to constrain the excess absorption as being due to intervening gas in the Milky Way and to estimate a total time-averaged column density of $N_{\mathrm{H}}=(7.4 \pm 0.1) \times 10^{21} \mathrm{~cm}^{-2}$. What is the origin of the extra Galactic neutral absorption of $N_{\mathrm{H}}=4.4 \times 10^{21} \mathrm{~cm}^{-2}$ with respect to the atomic $\mathrm{H}$ I value?

3C 111 is located at a relatively low latitude $\left(b=-8^{\circ} .8\right)$ with respect to the Galactic plane and it is known to lie behind the giant Taurus molecular cloud, which is the nearest large star-forming region in our Galaxy, at an estimated distance of about $200 \mathrm{pc}$ from us (Ungerer et al. 1985; Ungerechts \& Thaddeus 1987). Reynolds et al. (1998) suggested that this cloud complex may cause variations between the actual Galactic absorption along our line of sight and that inferred from $21 \mathrm{~cm}$ radio measurements due to inhomogeneities or the presence of molecular hydrogen.

Temporal variability in the strength and profile of the $4.83 \mathrm{GHz} \mathrm{H}_{2} \mathrm{CO}$ absorption line in radio observations shows that the cloud complex contains inhomogeneities on sub-parsec scales, and that between 30 and 100 clumps may lie along the line of sight to 3C 111 (Marscher et al. 1993; Moore \& Marscher 1995). A column density change is also marginally evident comparing the X-ray spectra performed a decade apart in Section 2.1. These inhomogeneities could in principle partially explain the discrepancy in column density estimates, but 3C 111 does not appear to be blocked by one or more particularly dense clumps (Reynolds et al. 1998).

Molecular hydrogen along our line of sight to 3C 111 associated with the Taurus molecular cloud may explain the excess. In fact, this gas would not contribute to the $21 \mathrm{~cm}$ emission, but the metals/dust associated with it would have opacity in the X-ray band. If about $60 \%$ of the gas along the line of sight to $3 \mathrm{C} 111$ is in molecular form rather than atomic, the discrepancy between the $21 \mathrm{~cm}$ radio measurements and the $\mathrm{X}$-ray could be resolved.

Pineda et al. (2010) showed a linear relationship between CO and extinction $A_{V}$ in the Taurus molecular cloud. Their results allowed for the estimation of the column of molecular hydrogen $\mathrm{H}_{2}$ with respect to the extinction: $N_{\mathrm{H}_{2}}=9.4 \times 10^{20} A_{V}$. The extinction along the line of sight to $3 \mathrm{C} 111$ is $^{7} 4.53$, which gives a molecular column density of $N_{\mathrm{H}_{2}} \simeq 4.3 \times 10^{21}$. This value is indeed consistent with the excess absorption with respect to the atomic HI. This is also consistent with the more general relation between the total column density of $\mathrm{HI}$ plus $\mathrm{H}_{2}$ reported in Bolatto et al. (2013) of $N_{\mathrm{H}} \simeq 1.9 \times 10^{21} A_{V} \mathrm{~cm}^{-2}$, which provides an estimate of $N_{\mathrm{H}} \sim 8 \times 10^{21} \mathrm{~cm}^{-2}$ toward 3C 111 . Finally, this is overall in agreement with the molecular hydrogen column density of $N_{\mathrm{H}_{2}} \simeq 4.5 \times 10^{21}$ molecules $\mathrm{cm}^{-2}$ estimated from the interstellar $\mathrm{CO}$ emission line measurement derived by

\footnotetext{
7 From https://ned.ipac.caltech.edu/.
}

Bania et al. (1991) toward 3C 111. However, we note that the cloud in front of $3 \mathrm{C} 111$ is translucent rather than opaque, and the $\mathrm{H}_{2}$ to $\mathrm{CO}$ ratio is better constrained for opaque clouds.

\section{Conclusions}

In this paper we show that the cold absorption detected in the $\mathrm{X}$-ray band toward 3C 111 is indeed of Galactic origin and it is very likely due to a combination of atomic and molecular gas. Therefore, we recommend the use of the total Galactic column density estimated here to be $N_{\mathrm{H}}=(7.4 \pm 0.1) \times 10^{21} \mathrm{~cm}^{-2}$ when studying the radio galaxy $3 \mathrm{C} 111$. For instance, a recent spectral energy distribution study of the radio, optical, IR, and X-ray knots in the extended jet of 3C 111 was found to be dependent on the assumed Galactic column (Clautice et al. 2016). On the other hand, the presence of these complexities toward 3C 111 make this object a very promising background source for multiwavelength studies of the characteristics of the atomic and molecular gas in the Taurus molecular cloud, which is the closest large star-forming region in our Galaxy (e.g., Ungerer et al. 1985; Marscher et al. 1993; Moore \& Marscher 1995; Güdel et al. 2007). The synergy between future deeper high-energy resolution X-ray observations and multiwavelength campaigns will allow us to investigate in more detail the characteristics of the complex multiphase medium in the Milky Way, and constrain the composition, elemental abundance, distribution, and the presence of dust.

F.T. thanks A. Bolatto and A. Marscher for useful comments. F.T. and C.S.R. acknowledges support for this work by the National Aeronautics and Space Administration (NASA) through Chandra Award Number GO4-15103A issued by the Chandra X-Ray Observatory Center, which is operated by the Smithsonian Astrophysical Observatory for and on behalf of NASA under contract NAS8-03060. F.T. acknowledges partial support by the Programma per Giovani Ricercatori-anno 2014 "Rita Levi Montalcini." E.B. is supported by the European Unions Horizon 2020 research and innovation programme under the Marie Sklodowska Curie grant agreement No. 655324, and by the ICORE program of the Planning and Budgeting Committee (grant No. 1937/12).

\section{References}

Asplund, M., Grevesse, N., Sauval, A. J., \& Scott, P. 2009, ARA\&A, 47, 481 Ballo, L., Braito, V., Reeves, J. N., Sambruna, R. M., \& Tombesi, F. 2011, MNRAS, 418, 2367

Bania, T. M., Marscher, A. P., \& Barvainis, R. 1991, AJ, 101, 2147 Bolatto, A. D., Wolfire, M., \& Leroy, A. K. 2013, ARA\&A, 51, 207 Bordoloi, R., Fox, A. J., Lockman, F. J., et al. 2017, ApJ, 834, 191 Braito, V., Reeves, J. N., Sambruna, R. M., \& Gofford, J. 2011, MNRAS, 414, 2739

Chatterjee, R., Marscher, A. P., Jorstad, S. G., et al. 2011, ApJ, 734, 43

Clautice, D., Perlman, E. S., Georganopoulos, M., et al. 2016, ApJ, 826, 109 Dickey, J. M., \& Lockman, F. J. 1990, ARA\&A, 28, 215

Dieter, N. H., Welch, W. J., \& Romney, J. D. 1976, ApJL, 206, L113

Eracleous, M., \& Halpern, J. P. 2003, ApJ, 599, 886

Faison, M. D., Goss, W. M., Diamond, P. J., \& Taylor, G. B. 1998, AJ, 116, 2916

Fox, A. J., Bordoloi, R., Savage, B. D., et al. 2015, ApJL, 799, L7

Gatuzz, E., García, J., Kallman, T. R., Mendoza, C., \& Gorczyca, T. W. 2015, ApJ, 800, 29

Gofford, J., Reeves, J. N., McLaughlin, D. E., et al. 2015, MNRAS, 451, 4169

Grandi, P., Torresi, E., \& Stanghellini, C. 2012, ApJL, 751, L3

Güdel, M., Briggs, K. R., Arzner, K., et al. 2007, A\&A, 468, 353

Kalberla, P. M. W., Burton, W. B., Hartmann, D., et al. 2005, A\&A, 440, 775 
Kataoka, J., Stawarz, Ł., Takahashi, Y., et al. 2011, ApJ, 740, 29

Kellermann, K. I., Sramek, R., Schmidt, M., Shaffer, D. B., \& Green, R. 1989, AJ, 98, 1195

Lewis, K. T., Eracleous, M., Gliozzi, M., Sambruna, R. M., \& Mushotzky, R. F. 2005, ApJ, 622, 816

Linfield, R., \& Perley, R. 1984, ApJ, 279, 60

Liszt, H., \& Lucas, R. 1996, A\&A, 314, 917

Liszt, H. S., \& Lucas, R. 1998, A\&A, 339, 561

Lohfink, A. M., Reynolds, C. S., Jorstad, S. G., et al. 2013, ApJ, 772, 83

Lohfink, A. M., Ogle, P., Tombesi, F., et al. 2015, ApJ, 814, 24

Marscher, A. P., Moore, E. M., \& Bania, T. M. 1993, ApJL, 419, L101

Moore, E. M., \& Marscher, A. P. 1995, ApJ, 452, 671

Nicastro, F., Senatore, F., Krongold, Y., Mathur, S., \& Elvis, M. 2016, ApJL, 828, L12

Ogle, P. M., Davis, S. W., Antonucci, R. R. J., et al. 2005, ApJ, 618, 139

Pineda, J. L., Goldsmith, P. F., Chapman, N., et al. 2010, ApJ, 721, 686

Reeves, J. N., Sambruna, R. M., Braito, V., \& Eracleous, M. 2009, ApJL, 702, L187

Reynolds, C. S., Iwasawa, K., Crawford, C. S., \& Fabian, A. C. 1998, MNRAS, 299, 410

Sambruna, R. M., Eracleous, M., \& Mushotzky, R. F. 1999, ApJ, 526, 60

Sambruna, R. M., Tombesi, F., Reeves, J. N., et al. 2011, ApJ, 734, 105
Schulz, N. S., Cui, W., Canizares, C. R., et al. 2002, ApJ, 565, 1141

Tombesi, F., Cappi, M., Reeves, J. N., et al. 2010a, A\&A, 521, A57

Tombesi, F., Sambruna, R. M., Reeves, J. N., et al. 2010b, ApJ, 719, 700

Tombesi, F., Sambruna, R. M., Reeves, J. N., Reynolds, C. S., \& Braito, V. 2011, MNRAS, 418, L89

Tombesi, F., Sambruna, R. M., Marscher, A. P., et al. 2012, MNRAS, 424, 754

Tombesi, F., Reeves, J. N., Reynolds, C. S., García, J., \& Lohfink, A. 2013, MNRAS, 434, 2707

Tombesi, F., Tazaki, F., Mushotzky, R. F., et al. 2014, MNRAS, 443, 2154

Tombesi, F., Reeves, J. N., Kallman, T., et al. 2016, ApJ, 830, 98

Tombesi, F., Mushotzky, R. F., Reynolds, C. S., et al. 2017, arXiv:1703.00516

Torresi, E., Grandi, P., Longinotti, A. L., et al. 2010, MNRAS, 401, L10

Torresi, E., Grandi, P., Costantini, E., \& Palumbo, G. G. C. 2012, MNRAS, 419,321

Ungerechts, H., \& Thaddeus, P. 1987, ApJS, 63, 645

Ungerer, V., Nguyen, Q.-R., Mauron, N., \& Brillet, J. 1985, A\&A, 146, 123

Urry, C. M., \& Padovani, P. 1995, PASP, 107, 803

Vermeulen, R. C., \& Cohen, M. H. 1994, ApJ, 430, 467

Willingale, R., Starling, R. L. C., Beardmore, A. P., Tanvir, N. R., \& O'Brien, P. T. 2013, MNRAS, 431, 394

Wilms, J., Allen, A., \& McCray, R. 2000, ApJ, 542, 914 